\title{
THE WAVE RESISTANCE FORMULA OF J.H. MICHELL (1898) AND ITS SIGNIFICANCE TO RECENT RESEARCH IN SHIP HYDRODYNAMICS
}

\author{
E. O. TUCK ${ }^{1}$
}

(Received 28 July 1988; revised 26 August 1988)

\begin{abstract}
John Henry Michell (1863-1940) published scientific papers only between 1890 and 1902, but included in his 23 papers from that short but productive period are some of the most important contributions ever made by an Australian mathematician. In this article I shall concentrate on the extraordinary 1898 paper "The wave resistance of a ship," Phil. Mag.(5) 45, 106-123. There are many reasons why this paper was an astounding achievement, but perhaps the most remarkable is that the resulting formula has not been improved upon to this day. In the computer age, many efforts have been made to do so, but with little success so far. The formula itself involves a triple integral of an integrand constructed from the offset data for the ship's hull, and even the task of evaluating this triple integral is not a trivial one on today's computers; another reason for admiration of Michell's own heroic hand-calculated numerical work in the 1890 's. Lack of a routine algorithm for Michell's integral has inhibited its use by naval architects and ship hydrodynamic laboratories, and there has been a tendency for it to receive a bad press based on unfair comparisons, e.g. comparison of model experiments (themselves often suspect) with inaccurate computations or computations for the wrong bull, etc. The original integral is in fact quite reasonable as an engineering tool, and some new results confirming this are shown. Improvement beyond Michell is however needed in some important speed ranges, and indications are given of recent approaches that may be promising.
\end{abstract}

\section{Historical summary}

J.H. Michell retired as Professor of Mathematics at the University of Melbourne in 1928, and died in 1940 at age 76 . His only formal publication after 1902 (a year in which he was elected a Fellow of the Royal Society at the early age of 39) was

\footnotetext{
${ }^{1}$ Applied Mathematics Department, University of Adelaide, S.A. 5001, Australia.

(C) Copyright Australian Mathematical Society 1989, Serial-fee code 0334-2700/89
} 
a calculus textbook that did not appear until after his retirement. Appendix 1 is a list of Michell's publications. Further details of his career and publications are available in [11], in which is also re-published the Royal Society obituary written by his brother A.G.M. Michell. A short biography by T.M. Cherry appears in the Australian Dictionary of Biography [3]. Incidentally, the brothers Michell are sometimes confused, especially by the engineering community, who know of A.G.M. Michell's thrust-bearing and other lubrication work, and assume that all work in elasticity and hydrodynamics of engineering relevance under the Michell name must be by the same person. However, unlike his brother, J.H. Michell was not an engineer, but first and always a mathematician. That is surprise enough; that he was also first and always an Australian is almost inconceivable to some.

Indeed, J.H. Michell seems to have spent all but about 6 years of his life in or near Melbourne. His only period away from Australia involved postgraduate study at Cambridge in 1884-1890, but that period was spent on Tripos-type coursework. His research career is a totally Australian one.

That it was such a short research career is a great Australian scientific tragedy, the cause for which seems not at all clear. Was he really burnt out as far as research was concerned at age 39 ? Or rather did the lack of acceptance (even of notice!) of his work by the practical world so disappoint him that he refused to publish any more of his research? Was there an argument about priority of ideas that turned him off the hurly-burly of the publishing game? I have not been able to answer these questions.

His brother gives a little information in the obituary. Statements like "... took his work as teacher very seriously and gave to it almost the whole of his time and mental energy", and "... onerous academic work almost excluded the possibility of continuously prosecuting original research" have a familiar ring to anyone who has ever sat on a promotion committee, but (although undoubtably accurate at least for his later years, as confirmed to me by recollections of former students) surely describe in part the consequences of abandonment of research activity, not its cause. In my opinion, a much more telling remark made by A.G.M. about J.H. is that "... absence of any response to such a paper as the 'Ship Waves' inevitably discouraged the making, or at least the publication, of investigations involving, for him, so much anxious labour."

I now turn to that particular paper, mainly because it is of prime interest to me, but also because it was perhaps Michell's finest achievement. For such an achievement to be ignored for a quarter of a century would be almost enough to cause anyone to give up research in disgust!

Before discussing the paper itself, I should report on my lack of success in discovering historical facts about its publication record. Naïvely, I thought that there might be archival material involving referee reports or the like. Sadly, no such material seems to have survived. The Philosophical Magazine was (and still 
is) published independently of any scientific society, by the London publishers Taylor and Francis, whom I visited in May 1986. They have deposited some archival material about their journal from that period in the St. Brides Publishing Library, Fleet Street, but this appears to consist of mainly financial details. Lord Kelvin was Editor at the time, but there is no Michell correspondence in the Kelvin papers at Glasgow University. The Royal Society has retained in its archives no documents relating to Michell's election as a Fellow in 1902, for which this paper must have been used as evidence.

There is a Michell file in the archives of Melbourne University, which contains some interesting handwritten notes and calculation sheets for other papers, but not for this one; I would very much (see below) have liked to have seen the notes for his numerical computation of the ship wave resistance example. The file does contain a set of hand-marked proofs of the ship paper, which is interesting for at least the fact that some of Michell's corrections were not implemented. In particular, he asked the printers to use partial derivative $(\partial)$ symbols, but the paper as published does not use them.

Such frustrating lack of archival material on journal publication is something any of us involved in editorial functions should see does not happen again. It has been the practice of the Australian Mathematical Society to deposit all correspondence regarding accepted papers in the archives of the Australian Academy of Science, so if we publish a masterpiece equivalent to Michell's, a future historian will have less difficulty than I did!

I must close this section with a sad quote of Michell from page 111 of the paper at issue, namely "... similar work to that of the present paper gives a theory of the damping of the oscillation of ships due to wave-making. This I hope to give in a subsequent paper." Of course, he never did. This problem was not solved, or at least a solution was not published, for nearly another half-century, e.g. by Havelock [7], although only even later, in work of Newman [10] and others, was a thin-ship method "similar" to Michell's used for the ship oscillation problem with non-zero forward speed.

\section{Summary of the ship wave paper}

As part of the obituary in [11], a brief appreciative summary of the ship wave paper by T.M. Cherry is quoted, but I now propose to highlight here some important modern aspects. The paper is concise and to the point, occupying 17 small pages in total, the main formula being derived within the first 8 pages. The paper reads like a modern research article, apart from the lack of partial derivative notation, which (as noted above) Michell wanted but did not get from the printers. 


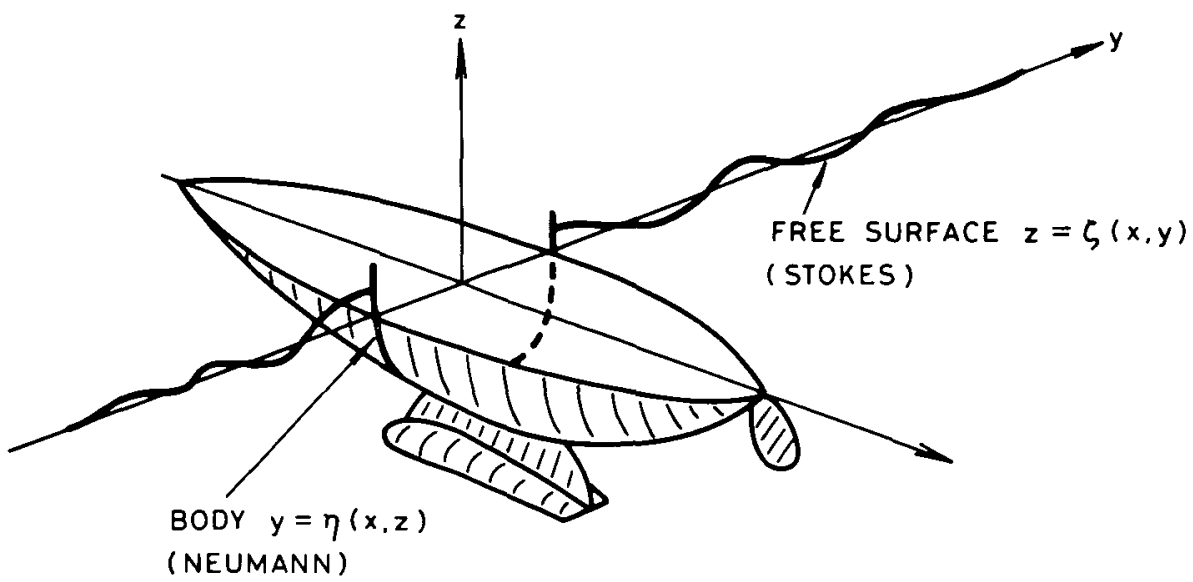

FiguRE 1. The fully nonlinear (Neumann-Stokes) boundary-value problem for Laplace's equation in the fluid domain exterior to a ship bull.

The problem is simply to obtain that portion of the drag force on a steadily moving ship which is due to the loss of energy into its wave pattern. Hence viscosity is neglected, this being justified by arguments pioneered by Froude for separating wave and viscous drag, but in Michell's own introductory words by boundary-layer-like arguments that could be said to anticipate Prandtl's by a decade or more. Once viscosity is neglected, classical inviscid-fluid theory indicates that the flow is irrotational almost everywhere, and a velocity potential exists.

The task of determining the velocity potential $\phi(x, y, z)$ is then a boundaryvalue problem for Laplace's equation

$$
\phi_{x x}+\phi_{y y}+\phi_{z z}=0
$$

in $|y|>\eta, z<\varsigma$, as sketched in Figure 1. It could be called a "Neumann-Stokes" boundary-value problem. That is, the boundary conditions in $y>0$ include the usual Neumann condition

$$
\phi_{y}=\phi_{x} \eta_{x}+\phi_{z} \eta_{z}
$$

of vanishing normal derivative on the ship's hull $y=\eta(x, z)$, representing the requirement that the fluid velocity be tangent to that surface. It is assumed that the problem is symmetric about the centerplane $y=0$ of the ship; incidentally, no-one has yet solved the generalisation of Michell's problem where this is not so, in spite of the fact that it has considerable interest for such problems as yawed yachts. In addition, as in nonlinear Stokes waves, a pair of boundary conditions must be satisfied on the (unknown) free surface $z=\varsigma(x, y)$ of the water, namely a kinematic condition

$$
\phi_{z}=\phi_{x} \zeta_{x}+\phi_{y} \zeta_{y}
$$


again expressing flow tangency, and a dynamic condition

$$
2 g \varsigma+\phi_{x}^{2}+\phi_{y}^{2}+\phi_{z}^{2}=U^{2}
$$

expressing (via Bernoulli's theorem) constancy of pressure. Here $g$ is the (constant) acceleration of gravity and $U$ the ship's speed. The problem formulation is completed by suitable boundary conditions at infinity, including a "radiation condition" that demands that any waves lie behind, not ahead of the ship.

This Neumann-Stokes problem is nonlinear, due to the quadratic nature of the Bernoulli free-surface condition, and also rendered difficult by the fact that this free surface $z=\zeta(x, y)$ is not known in advance. So difficult that there have been no successful numerical solutions of it to this day.

Michell then fully linearises the Neumann-Stokes problem, such linearisation (of both body and free surface conditions) being justified on the basis that the ship is thin, i.e. that its longitudinal slope is everywhere numerically small. The linearised hull boundary condition (on $y=0$ ), namely

$$
\phi_{y}=U \eta_{x}
$$

may be called a Michell condition, and the linearised free-surface condition (on $z=0$ ), namely

$$
g \phi_{z}+U^{2} \phi_{x x}=0
$$

called a Kelvin condition. The resulting "Michell-Kelvin" boundary-value problem has several simplifying features, not least of which is that the only boundaries are planes $y=0$ and $z=0$. Michell makes no further approximations, and proceeds to solve the Michell-Kelvin problem exactly using Fourier-transform methods.

The role played by the depth of the water in Michell's paper is a little mixed. The boundary-value problem is at first formulated for a body of water of finite uniform depth, and the special form of the Fourier representation that Michell needs is derived for such a case. However, he then assumes after 7 pages ".. for simplicity, the water infinitely deep", and indeed that simplicity is evident, for he takes only one further page to derive the wave resistance formula for the infinite-depth case. The equivalent wave resistance formula for finite depth was in fact not derived [12] for another 38 years.

However, a little-known feature of the ship paper is that, almost as an afterthought, Michell also includes on its last two pages a mini-study of the shallowwater case, i.e. of the limit as the water depth vanishes, that is quite independent of his finite-depth work from earlier in the paper. This shallow-water analysis is itself of remarkable historical interest, since it uses what we would now identify as an analogy between sub- or super-critical hydraulics, and sub- or super-sonic aerodynamics. Since manned flight was still a decade away, and supersonic flight 
a half century away, this is truly remarkable work, that would make these two pages significant even if the infinite-depth portion were discounted.

However, it must be said that quite similar and now better known aerodynamic work was being done by Joukowski at about that time (though published later, see e.g. [8]). Michell gives no indication that he has seen any of Joukowski's work, and it hardly seems likely in view of his geographical isolation that he had done so. At the very least, Michell's application of the hydraulic analogy to the ship context is original, and personally I believe that (based on nothing more than these two pages) he should be given at least equal credit with Joukowski for this whole research area.

Returning to the main topic of the Michell paper, namely the infinite-depth wave resistance, Michell uses his special Fourier-integral theorem (derived as the limit of a Fourier series, and then proved directly) to obtain the velocity potential, and hence the pressure on the limiting plane $y=0$ representing the linearised hull, and integrates to yield the final formula, namely (almost as it appears on his p.113):

$$
R=\frac{4 \rho g^{2}}{\pi U^{2}} \int_{1}^{\infty}\left(I^{2}+J^{2}\right) \frac{\lambda^{2} d \lambda}{\sqrt{\lambda^{2}-1}}
$$

where

$$
I=\iint_{H} \eta_{x}(x, z) e^{\lambda^{2} g z / U^{2}} \cos \left(\lambda g x / U^{2}\right) d x d z
$$

with a similar integral for $J$ involving sine instead of cosine.

This is the famous Michell integral. It must have frightened some of the practical people that Michell hoped would use it, since he first derived it as a quintuple integral. However, the final form, as quoted here, shows it to be "only" a triple integral. One must first evaluate the quantities $I$ and $J$, by a double integral over the projection $H$ of the hull surface onto the centreplane $y=0$, and afterward integrate a function involving the squares of these quantities, with respect to a variable $\lambda$. Thus the formula provides the wave resistance as a positive definite quadratic functional in the input quantity $\eta(x, z)$ that defines the geometry of the hull.

Just this qualitative feature alone of the formula is pioneering. I have heard it asserted that this was the first ever "engineering design" formula, in the sense that it provides an output quantity of design interest from an input that specifies the actual (unrestricted) design geometry. Certainly in hydrodynamics before Michell, the only solutions of boundary-value problems were for special shapes like circles and spheres. Michell's theory is quite similar to that for (nonlifting) thin wings in aerodynamics, but that theory (although very much simpler because of the absence of the free surface) took another 20 years to be developed, and of course when it appeared there was no credit given to Michell. 
Having derived the formula, Michell goes on to prove explicitly that the resistance vanishes at high speed, i.e. $R \rightarrow 0$ as $U \rightarrow \infty$, and to derive some other properties of the solution in terms of Bessel functions. He then, quite remarkably, and in less than one page, provides a specific example involving a numerical evaluation of the integral. I reproduce that particular page as Appendix 2, since it also contains clear indications of his (unrealised) expectations for acceptance of his formula in the practical world.

Michell does not say what "mechanical quadrature" he used. The integral with respect to $\lambda$ has a square-root singularity at $\lambda=1$, which should be removed by a transformation such as $\lambda=\sec \theta$. Incidentally, a modern treatment (e.g. [16]) of this type of integral identifies $\theta$ as the angle at which the wave energy is propagating. The final integral then has a nice physical interpretation as a summation of contributions from waves of all possible angles.

Perhaps Michell did not make such a transformation before using his mechanical quadrature. In any case, my own recomputation of the actual integral quoted by Michell as 0.620 yielded the slightly more accurate value 0.6157 ; I used Simpson's rule after the $\sec \theta$ transformation, and needed to divide the interval $0<\theta<\pi / 2$ into at least 50 sub-intervals, since the integrand is very oscillatory. This computation was also confirmed by inputting this special hull to a general-purpose program [15] that takes as input a table of the offsets $\eta(x, z)$.

So Michell did not quite achieve the 3-figure accuracy that he quoted. We must forgive him for this. He did get about 2 figures, his error being at most $0.7 \%$. Just think of the effort he must have expended to achieve this, not having available even the most rudimentary of computing equipment! The integrand won't even fit on one line of the page, it is so complicated. When he first saw it, I am sure he must have despaired of ever being able to evaluate it numerically, but he did, and without blunder, to adequate engineering accuracy.

\section{Modern developments}

We must draw the shades on the next 25 years. Did Michell expect the naval architectural world to receive his wonderful formula instantly as its salvation? He had some right to, but it didn't. Sir Thomas Havelock [7] was the most famous theoretician working in ship hydrodynamics in the period, and he had already published several papers on the wave resistance problem (the first in 1909) before making explicit use of Michell's integral in 1923. It seems likely that (in spite of the fact that it was published in one of the world's premier scientific journals) neither Havelock nor almost anyone else had even noticed it until then. Havelock's outstanding achievements were spread over 60 papers 


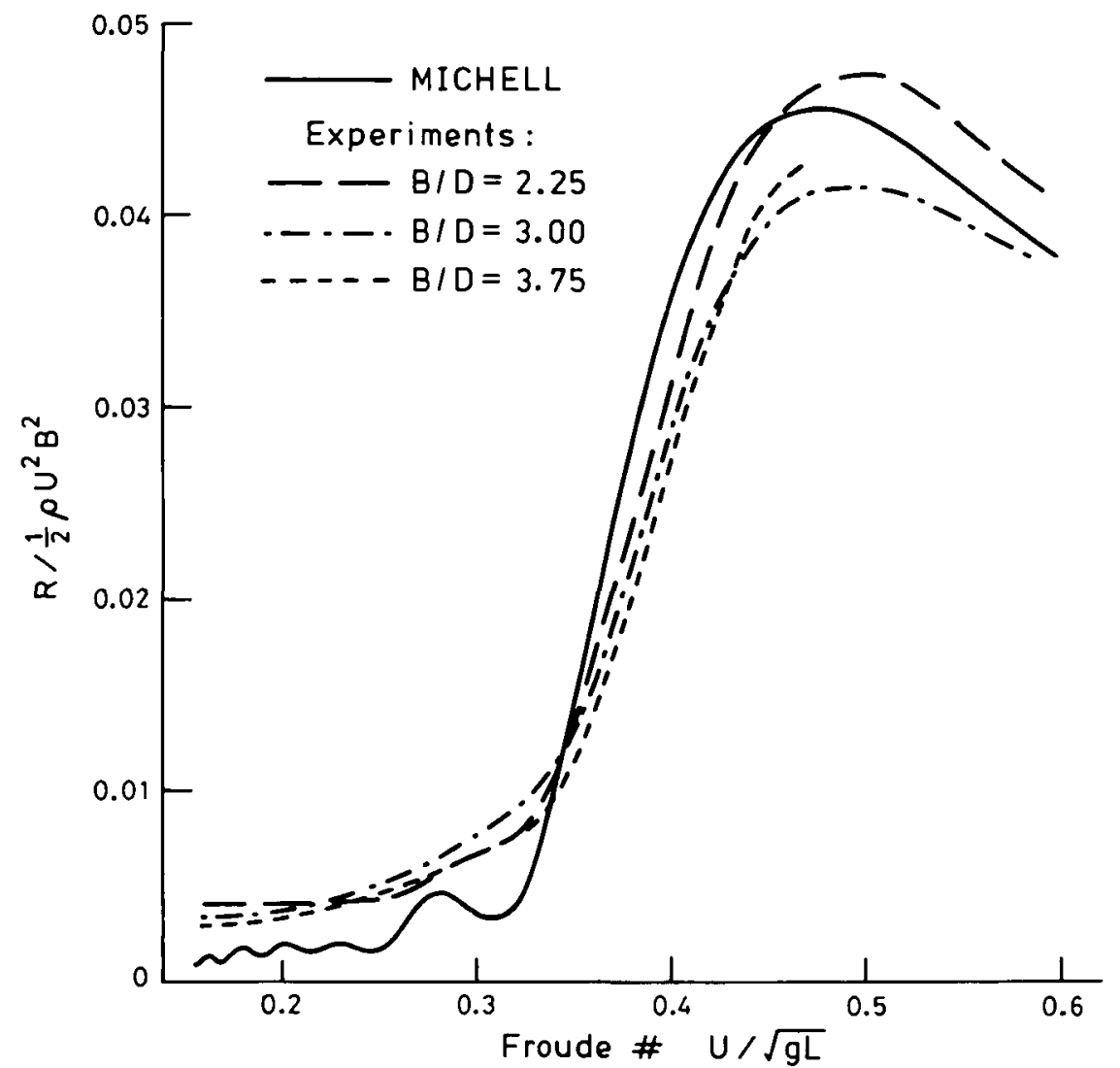

FIGURE 2. Wave resistance of a "Taylor Standard Series" ship, with prismatic coefficient 0.56 and length/draft ratio 30 .

in a 40-year career. The contrast with Michell's solitary paper on this topic is interesting.

C. Wigley [18] was an advocate of use of Michell's formula from 1925 onward, and Wigley and Havelock then provided sample computations and comparison (both favourable and unfavourable) with experiment. It is appropriate at this point to provide a new example of such comparisons; many more are provided by Wehausen [16] and others.

Figure 2 shows the wave resistance of a conventional merchant-ship hull as a function of speed, for three different beam/draft ratios. The experimental results at beam/draft $=2.75$ and 3.75 are from D.W. Taylor's original model data, as tabulated by Gertler [5], while those for beam/draft $=3.00$ are as retested by Graff et al [6].

A feature of Michell's integral is that it scales with the square of the ship's beam, so that the nondimensional resistance coefficient shown in Figure 2, (with 
beam-squared in the denominator) should be independent of the beam. The three experimental curves are for ships of the same shape, length and draft, but varying beam. Hence if Michell was right, all three curves would agree with each other and all would agree with computations from Michell's integral. Further, since Michell assumes small beam, Michell should agree best with the experiments (long dashes) for the model with the least beam.

Looked at as a whole, one would have to say that the formula is doing rather well, as a predictor of the wave resistance over a complete speed range including that for which the resistance is a maximum. The error is generally less than that to be expected in the experiments, e.g. that due to scale effects from use of different model sizes.

Unfortunately, the naval architect is not really interested in the complete graph of wave resistance, and in particular (for very good reasons!) is not usually interested in those speeds where the wave resistance is a maximum. Commercially viable vehicles run at speeds just below those where the wave resistance begins to increase toward its main maximum. That increase is so rapid that it is not (except in some military contexts) sensible to provide the engine power to overcome it. This means that the important speed range is for a Froude number (nondimensional speed) of about 0.2 to 0.35 .

Michell's integral does not do a particularly good job in that range. The errors are of the order of $50 \%$ to $100 \%$. In particular, the last minimum, at a Froude number of about 0.31 in Figure 2, is not observed in practice. Incidentally, Figure 2 indicates an aspect of the model-testing methodology that worries some observers, namely that the experimental "wave resistance" coefficient does not tend to zero as the speed tends to zero. Furthermore (see the discussion to [6]) the anomalous zero speed limit changes its value when models of different sizes are tested. The magnitude of this effect is comparable with the errors between Michell's integral and the experiments; however, it would not be satisfactory to simply subtract the zero-speed limit from the experimental results, since this would make the agreement worse at higher speeds.

Really, what Michell is saying is that (relative to the peak resistance, and also therefore relative to the viscous drag), the wave resistance is quite small in the speed range corresponding to Froude numbers less than 0.3. But that is little comfort to the naval architect, who is anxious to reduce it to the lowest possible value, and hence wants an accurate predictor of what little he has left.

The fact that Michell's integral is only moderately successful at practical speeds gradually became clear in the years after its rediscovery by Wigley and Havelock, especially as precomputer evaluation techniques were developed by authors such as Weinblum [17]; see also Birkhoff et al [2]. Even so, its routine evaluation as a triple integral for actual hull data (rather than idealised 
mathematically-defined surfaces) was hardly feasible until the advent of computers. Even now, very few serious attempts have been made to provide such code. There are important numerical issues with physical (short-wave) and geometrical (end-effect) implications concerning accuracy of the integration to determine $I$ and $J$ when the parameter $\lambda$ is large. An implementation of my own [15] uses Filon's quadrature for this purpose.

But however carefully Michell's integral is worked out, and however little faith one has in the model experiments (which are not easy in a speed range where viscosity dominates the measured output and the "experimental" wave resistance must be estimated as the difference of two larger numbers), there seems little doubt that a better theory is needed in this speed range. No such theory yet exists.

It is clear that Michell's thin-ship assumption $\eta_{x}<<1$ is the culprit, and must be relaxed. In the age of super-computers, one might expect that a direct attack on the original nonlinear boundary-value problem of Figure 1 would be feasible. Perhaps so, but we are still a long way from that. In the meantime, there has been some work on the so-called "Neumann-Kelvin" problem, namely that in which one retains the full Neumann boundary condition on the actual hull surface $y=\eta(x, z)$, while (inconsistently) simplifying the full nonlinear Stokes free-surface boundary condition to that of Kelvin, by linearisation onto the plane $z=0$. Even then, one is faced with quite intimidating numerical difficulties, and some authors [4] have expressed pessimism about the feasibility of routine numerical solution of the Neumann-Kelvin problem.

There is even some doubt about existence, in the formal mathematical sense, of a solution to the Neumann-Kelvin problem! After all, if the ship is not thin, what justification do we have for linearisation of one boundary condition without the other? The Neumann and Kelvin boundary conditions are incompatible at their junction, a defect from which neither the fully nonlinear Neumann-Stokes problem, nor the fully linearised Michell-Kelvin problem appears to suffer. This is an aspect of the ship-hydrodynamic problem that could stand some puremathematical attention.

There are some other promising recent research directions, all associated with exploiting the fact that most ships are not only thin, but also slender, in the sense that their draft as well as their beam is small. Generally these slender ship theories retain some nonlinear features of both boundary conditions, but achieve tractable numerical properties by replacing the 3D Laplacian by a 2D Laplacian in each separate cross-section plane. There were several rather unsuccessful attempts at such theories in the 1960s, including one of my own [13], but quite recently $[9,14]$ a new look at this class of approximation has seemed likely to allow better predictive capability in the important speed range. 
Meanwhile, the original unadulterated Michell integral remains competitive and reliable. After reviewing the performance of some 22 rival computer programs, all complex in detail and expensive in implementation, Bai [1] was forced to conclude in 1979 that "... wave resistance predictions by first-order thin-ship [i.e. Michell] theory are rather consistent in comparison with experimental data and not worse than the envelope of predictions of seemingly more sophisticated methods ...". The situation is not much better today.

\section{Acknowledgement}

I acknowledge gratefully many critical comments on this article, received both from those with memories of Michell, and from those with views on the technical ship hydrodynamic matters discussed. Although I have incorporated into the final version as much information as possible drawn from these comments, it remains to a large extent a personal view of the status of Michell's work.

\section{References}

[1] K.J. Bai and J.H. McCarthy (eds.), Proc. workshop on ship wave resistance computations, David W. Taylor N.S.R.D.C., Bethesda, Md., November 1979.

[2] G. Birkhoff, B.V. Korvin-Kroukovsky and J. Kotik, "Theory of the wave resistance of ships. Part 1: The significance of Michell's integral", Trans. Soc. Nav. Architects Mar. Eng. 62 (1954) 359-371.

[3] T.M. Cherry, "J.H. Michell", Australian dictionary of biography 10 (1986) 494-495.

[4] L.J. Doctors and R.F. Beck, "Numerical aspects of the Neumann-Kelvin problem", $J$. Ship Res. 31 (1987) 1-13.

[5] M. Gertler, "A reanalysis of the original test data for the Taylor standard series", David Taylor Model Basin Rep. 806 (1954).

[6] W. Graff, A. Kracht and G. Weinblum, "Some extensions of D.W. Taylor's standard series", Trans. Soc. Nav. Architects Mar. Eng. 72 (1964) 374.

[7] T.H. Havelock, "The collected papers of Sir Thomas Havelock", edited by C. Wigley, Office of Naval Research, Washington D.C., 1963.

[8] N.E. Joukowski, "On the concomitant wave", in Collected Works of N.E. Zhukovskii Vol. 2, pp. 712-745, Moscow, 1949 (in Russian).

[9] H. Maruo, "Evolution of the theory of slender ships" Weinblum Memorial Lecture, U.S. National Academy of Sciences, April 1988.

[10] J.N. Newman, "The damping and wave resistance of a pitching and heaving ship", J. Ship Res., 3 (1959) 1-19.

[11] J.R.M. Radok and F.W. Niedenfubr, "The collected works of J.H. and A.G.M. Michell", Noordhoff 1964.

[12] L.N. Sretenskii, "A theoretical investigation on wave resistance", Trans. Tsent. AeroGidrodinam. Inst. 319 (1937) (in Russian).

[13] E.O. Tuck, "A systematic asymptotic expansion procedure for slender ships"' J. Ship Res. 8 (1964) 15-23. 
[14] E.O. Tuck, "A strip theory for wave resistance", Srd Int. Workshop on Water Waves and Floating Bodies, Wood's Hole, Massachusetts, April 1988.

[15] E.O. Tuck, "Wave resistance of thin ships and catamarans", University of Adelaide, Applied Mathematics Rep. T8701, January 1987.

[16] J.V. Wehausen, "The wave resistance of ships", Adv. in Appl. Mech. 13 (1973) 93-245.

[17] G. Weinblum, "A systematic evaluation of Michell's integral", David Taylor Model Basin Rep.886 (1955).

[18] W.C.S. Wigley, "Ship wave resistance. A comparison of mathematical theory with experimental results", Trans. Inst. Nav. Architects, 68 (1926) 124-137.

\section{Appendix 1: Publications of J.H. Michell}

1. "The small deformation of curves and surfaces with applications to the vibrations of a helix and a circular ring" Messeng. Math. 19 (1890) 68-82.

2. "On the exhaustion of Neumann's mode of solution for the motion of solids of revolution in liquids, and similar problems" Messeng. Math. 19 (1890) 83-86.

3. "Vibrations of a string stretched on a surface" Messeng. Math. 19 (1890) 87-88.

4. "On the stability of a bent and twisted wire" Messeng. Math. 19 (1890) 181-184.

5. "On the theory of free stream lines" Phil. Trans. A. 181 (1890) 389-431.

6. "On a property of algebraic curves" Australasian Assoc. Adv. Sci. Report (1892) 257.

7. "On the bulging of flat plates" Australasian Assoc. Adv. Sci. Report (1892) 258.

8. "The highest waves in water" Phil. Mag. (5) 36 (1893) 430-437.

9. "A map of the complex Z-function: a condenser problem". Messeng. Maths. 23 (1894) 72-78.

10. "The wave resistance of a ship" Phil. Mag. (5) 45 (1898) 106-123.

11. "On the direct determination of stress in an elastic solid, with application to the theory of plates" Proc. Lond. Math. Soc. 31 (1899) 100-124.

12. "The stress in a rotating lamina" Proc. Lond. Math. Soc. 31 (1899) 124-130.

13. "The uniform torsion and flexure of incomplete tores, with application to helical springs" Proc. Lond. Math. Soc. 31 (1899) 130-146.

14. "The transmission of stress across a plane of discontinuity in an isotropic elastic solid, and the potential solutions for a plane boundary" Proc. Lond. Math. Soc. 31 (1899) 183-192.

15. "Some elementary distributions of stress in three dimensions" Proc. Lond. Math. Soc. 32 (1900) 23-35.

16. "Elementary distributions of plane stress" Proc. Lond. Math. Soc. 32 (1900) 35-61.

17. "The stress in an aeolotropic elastic solid with an infinite plane boundary" Proc. Lond. Math. Soc. 32 (1900) 247-258.

18. "The stress in the web of a plate girder" Quart. J. Pure Appl. Math. 31 (1900) 377-382.

19. "The theory of uniformly loaded beams" Quart. Jour. of Math. 32 (1900) 28-42.

20. "The determination of the stress in an isotropic elastic sphere by means of intrinsic equations" Messeng. Math. n.s. 350 (1900) 16-25.

21. "The uniplanar stability of a rigid body" Messeng. Math. n.s. 351 (1900) 35-40.

22. "The inversion of plane stress" Proc. Lond. Math. Soc. 34 (1902) 134-142.

23. "The flexure of a circular plate" Proc. Lond. Math. Soc. 34 (1902) 223-228.

24. (with M.H. Belz) "The elements of mathematical analysis" (2 vols) Macmillan 1937. 


\section{Appendix 2: An extract from the wave-resistance paper}

As an illustration of the process of calculating the resistance of a given ship at any speed, we may consider one of simple analytical form which is fairly ship-shape and easily realisable. Experinental confirmation of the result was not practicable, and the matter must be left in the hands of those who bave the necessary apparatus at command.

Let the surface of the ship be

$$
y= \pm c(1+\cos a x)(1+\cos b z)
$$

between

$$
\begin{aligned}
& x= \pm \pi / a \\
z=0 & \text { and } \pi / b ;
\end{aligned}
$$

Fig. 2.

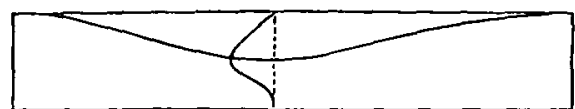

so that, for $y$ positive,

$$
f(x, z)=\frac{d y}{d x}=-a c \sin a x(1+\cos b z) .
$$

Here $I=0$,

$$
\text { and } \begin{aligned}
J & =-a c \int_{0}^{\pi / b}(1+\cos b z) e^{-\lambda^{2} z / k} d z \int_{-\pi / a}^{\pi / a} \sin a x \sin \lambda x / k d x \\
& =-a c \frac{k}{\lambda^{2}}\left(2 \lambda^{4}+b^{2} k^{2}-e^{-\pi \lambda^{2} / b k} b^{2} k^{2}\right) \frac{2 a k^{2}}{\left(\lambda^{4}+b^{2} k^{2}\right)\left(a^{2} k^{2}-\lambda^{2}\right)} \sin \pi \lambda / k a,
\end{aligned}
$$

where $k=v^{2} / g$; and therefore

$$
\mathrm{R}=\frac{16 g \rho}{\pi} a^{4} c^{2} k^{5} \int_{1}^{\infty}\left(2 \lambda^{4}+b^{z} k^{2}-e^{-\pi \lambda^{2} / b k} b^{2} k^{2}\right)^{2} \frac{\sin ^{2} \pi \lambda / k a}{\left(\lambda^{4}+b^{2} k^{2}\right)^{2}\left(a^{2} k^{2}-\lambda^{2}\right)^{2}} \frac{d \lambda}{\lambda^{2} \sqrt{\lambda^{2}-1}}
$$

which is best calculated by mechanical quadrature.

Suppose, for example, in foot-second units

$$
\begin{aligned}
v & =20 \text { (velocity of ship), } \\
2 \pi / a & =200 \text { (length of ship), } \\
\pi / b & =20 \text { (depth below water-line), } \\
8 c & =32 \text { (greatest breadth), }
\end{aligned}
$$

then the integral is found to be $\cdot 620$, and the resistance is

$$
\mathrm{R}=940 \text { lbs. wt. about. }
$$

This seems to be about what one would expect from the experimental results available; but I know of no formula with which to compare it, and experiment alone can decide whether the theory has numerical value. 